INVESTIGACIONES

\title{
LOS PROCESOS DE ENSEÑANZA-APRENDIZAJE EN EL AULA UNIVERSITARIA: UNA MIRADA DESDE LAS CREENCIAS DE ACADEMICOS DE TRABAJO SOCIAL Y PERIODISMO
}

\author{
The teaching-learning processes in the university classroom: \\ A glance from the beliefs of academics from social work and journalism
}

\author{
María Inés Solar R., Claudio Díaz L. \\ Universidad de Concepción. marsolar@udec.cl
}

\begin{abstract}
Resumen
El presente artículo tiene como finalidad presentar los primeros resultados del proyecto FONDECYT 1060622 "El sistema de creencias y prácticas pedagógicas del docente universitario y su implicancia en el proceso de enseñanza-aprendizaje". Este proyecto tiene como objetivo develar las creencias de 10 académicos sin formación pedagógica de origen, de la Octava Región del país, mediante una entrevista semiestructurada aplicada a 10 académicos de las áreas de Trabajo Social y Periodismo de una universidad del Consejo de Rectores de la ciudad de Concepción. Los resultados han sido presentados en ocho categorías referidas a las variables didácticas del proceso de enseñanza-aprendizaje. Las conclusiones, en primera instancia, parecen indicar que los académicos universitarios, a pesar de no poseer una formación pedagógica, sostienen creencias que se condecirían con prácticas de enseñanza de calidad, por lo menos a nivel de discurso.
\end{abstract}

Palabras clave: creencias, variables didácticas, educación superior.

\begin{abstract}
The present article aims at presenting the first results of the government-funded project 1060622 FONDECYT "El sistema de creencias y prácticas pedagógicas del docente universitario y su implicancia en el proceso de enseñanza-aprendizaje". This project aims at unravelling the beliefs of academics who did not receive any pedagogical preparation during their undergraduate training. This article presents the results from a semi-structured interview applied to 10 academics from the areas of Social Work and Journalism of a Chilean university in the city of Concepción. The results have been displayed in eight categories referred to by the didactic variables involved in the teaching-learning process. The preliminary conclusions seem to indicate that college professors, in spite of not having pedagogical preparation, possess beliefs that would match with their teaching practices and would lead to quality learning, at least at a discourse level.
\end{abstract}

Key words: beliefs, teaching components, tertiary education. 


\section{INTRODUCCION}

1.1. Los docentes y la comprensión de sus experiencias pedagógicas. Uno de los grandes desafíos de los docentes es encontrarle significado a su experiencia pedagógica. El rol de las teorías, las prescripciones externas y las experiencias de otros docentes adquiere relevancia cuando ellas pueden articularse con la experiencia del docente y, a su vez, tengan sentido en su trabajo. No en vano los formadores de docentes y los investigadores buscan comprender la forma como las instituciones educativas median y transforman el 'qué' y 'cómo' enseñan los docentes, con el fin de conocer sus procesos de aprendizaje. Un punto interesante aquí es la forma como dichos contextos pueden ser organizados de manera tal que apoyen el aprendizaje de los nuevos docentes y la transformación de las prácticas pedagógicas de los docentes experimentados (Andrés y Echeverri 2001; Appel 1995; Bailey, Curtis \& Nunan 2001).

El docente es alguien que está continuamente intentando aprender más sobre sí mismo y su manera de trabajar. Algunos autores han caracterizado la construcción del conocimiento de la enseñanza como "un vuelco paradigmático", argumentando que es tiempo de reconocer la esterilidad de la pretensión de un conocimiento científico y tecnológico que guíe la actuación de los profesionales, creando situaciones artificiales entre el conocimiento de unos y la práctica de otros, reproduciendo la dicotomía entre el conocimiento y la actuación en la práctica cotidiana (Biddle, Good \& Goodson 2000; Camps 2001; Cárdenas, Rodríguez y Torres 2000; Crookes 2003).

La investigación de los pensamientos y conocimientos de los profesores/as tiene la intención tanto de comprender las concepciones, creencias, dilemas, teorías que gobiernan la práctica profesional como identificar los procesos que constituyen el aprender a enseñar y las categorías conceptuales en las que se articula el conocimiento para desarrollar la actividad profesional de la enseñanza.

\section{LOS ESTUDIOS SOBRE EL PENSAMIENTO DEL PROFESORADO}

Una de las líneas de investigación que se ha desarrollado con mayor profusión en las últimas décadas en torno al conocimiento profesional es sin duda la del 'pensamiento del profesor', hasta el punto de que se habla incluso del 'paradigma del pensamiento del profesor'. El interés fundamental de los estudios desarrollados bajo este enfoque es conocer cuáles son los procesos de razonamiento que ocurren en la mente del docente durante su actividad profesional.

A comienzos de los setenta -durante el auge de la Psicología Cognitiva- aparecen nuevos enfoques como los estudios sobre el conocimiento práctico del docente. Esta línea de trabajo surge para paliar algunas de las carencias de los enfoques de procesamiento de información, es decir, ante el reduccionismo, la excesiva formalización y la descontextualización de los enfoques de procesamiento de la información emergen aproximaciones prácticas e integradoras, que defienden que el conocimiento que facilita la comprensión del contexto de actuación docente, y que determina en última instancia las decisiones y cursos de acción ejecutados durante la enseñanza, es un conocimiento personal o práctico-reflexivo, producto de la biografía y experiencias pasadas del docente, de sus conocimientos actuales y de su relación activa con la práctica. Es un saber hacer 
en su mayor parte tácito que se activa en la acción misma (Frabboni 2001; Gelhard \& Oprandy 1999; Gonzáles, Río y Rosales 2001; Johnson \& Golombek 2002).

Los docentes afrontan su actividad profesional mediante un sistema idiosincrásico de conocimientos que es producto de la elaboración personal que éstos hacen de sus ideas en un contexto institucional y social determinado. Dichos conocimientos son los que verdaderamente utiliza el docente y en "su conformación entran en juego factores subjetivos, biográficos y experienciales, así como aspectos objetivos contextuales" (Kansanen, Tirri, Meri, Krokfors, Huso \& Jyrhämä 2000; Libedinsky 2001; Menin 2001; Muchmore 2004).

El docente es, sin duda, un sujeto 'constructivista' que continuamente construye, elabora y prueba su teoría personal del mundo. Esto es consistente con la visión actual de la enseñanza considerada como una actividad del pensamiento profesional, en la cual el cambio conceptual debe ser reconocido como el centro del aprendizaje del docente. Las teorías personales se definen como un sistema en el cual subyacen constructos que el docente usa cuando piensa, evalúa, clasifica y guía su práctica pedagógica (RichardAmato 2003; Sanjurjo 2002; Tsui 2003; Woods 1996). Las teorías personales de los docentes responden casi siempre al sentido común y son de naturaleza tácita. No son necesariamente coherentes; sino que más bien se consideran como dinámicas y sujetas al cambio y a la reformulación gradual.

\section{III. ¿QUE SON LAS CREENCIAS?}

A diferencia de las creencias ideológicas más generales que pueden ser descontextualizadas y abstractas, las creencias del docente están relacionadas con situaciones específicas orientadas a la actuación e incluyen tanto las creencias que los docentes tienen sobre su trabajo (objetivos, concepciones de sus estudiantes, contenidos, etc.) como las "ways in which they [give] meaning to these beliefs by their behavior in the classroom" (Andrews 2003; Ben-Peretz 2002; Carusetta \& Cranton 2005; Freeman 2002).

Borg (2003) usa el término cognición del docente para referirse a las dimensiones cognitivas de la enseñanza no observables, es decir, lo que los docentes conocen, creen, y piensan. Esto significa que los docentes son agentes activos en la toma de decisiones instruccionales haciendo uso de complejas redes de conocimientos, pensamientos y creencias. Estas redes tienen las siguientes características: se orientan a la práctica, son personalizadas y son sensibles al contexto.

Las personas utilizan las creencias para recordar, interpretar, predecir y controlar los sucesos que ocurren y tomar decisiones. Ellas son producto de la construcción del mundo y se basan en procesos de aprendizaje asociativo; pero también tienen un origen cultural en tanto se construyen en "formatos de interacción social y comunicativa" (Goodson \& Numan 2002; Hamel 2003; Hashweh 2005; Johnstone 2000). Las creencias están compuestas por conjuntos más o menos integrados y consistentes de ideas que se construyen, a partir de las experiencias cotidianas. Son versiones incompletas y simplificadas de la realidad, que si bien permanecen inaccesibles a la conciencia, "tienen algún nivel de organización interna, estructuración y sistematicidad". 


\section{DISEÑO DE INVESTIGACION}

4.1. Pregunta de investigación. ¿Qué creencias tienen los académicos no pedagogos universitarios de las carreras de Trabajo Social y Periodismo de una universidad de la ciudad de Concepción acerca de la enseñanza y el aprendizaje?

4.2. Supuestos de esta investigación. Los siguientes son los supuestos que sustentan esta investigación:

- El docente es un sujeto reflexivo que experimenta situaciones de enseñanza-aprendizaje y les otorga significado.

- La reflexión lleva a la toma de decisiones.

- Un docente informado debería tener una base de conocimiento más profundo acerca de la enseñanza-aprendizaje.

- Se puede aprender mucho sobre la enseñanza-aprendizaje mediante el autoconocimiento.

4.3. Justificación de los supuestos. El profesor es un sujeto reflexivo, racional, que toma decisiones, emite juicios, tiene creencias y genera rutinas propias de su desarrollo profesional. Se acepta, además, que las creencias del docente guían y orientan su conducta. Los docentes no están frecuentemente conscientes del tipo de enseñanza que realizan o cómo ellos manejan muchas decisiones emergentes en el aula. Puesto que muchas cosas suceden simultáneamente durante una clase, es algunas veces difícil, para algunos docentes, estar conscientes de lo que allí sucede.

4.4. Objetivo general. Comprender las creencias de los académicos no pedagogos en torno al funcionamiento de las variables didácticas que intervienen en la enseñanza-aprendizaje en contextos universitarios

4.5. Metodología. La presente investigación corresponde a un estudio de carácter descriptivo-interpretativo, congruente con un diseño de estudio de caso, puesto que indaga en las creencias y actuaciones de los docentes universitarios en el contexto del proceso de enseñanza-aprendizaje. Se pretende realizar un análisis de la realidad socioeducativa mediante un proceso de indagación que se caracteriza por el examen detallado, comprehensivo, sistemático y en profundidad del caso objeto de estudio.

4.6. Sujetos informantes. Los académicos no pedagogos que participan en este estudio imparten docencia en una Universidad del Consejo de Rectores de la ciudad de Concepción y suman 10 en total. Estos docentes provienen de las áreas de Trabajo Social y Periodismo.

4.7. Técnica de recopilación de información. La técnica utilizada fue una entrevista semiestructurada cuyo objetivo era examinar las creencias de los docentes en torno a las variables y modelos didácticos que subyacen en la enseñanza-aprendizaje. 


\section{ANALISIS DE LOS DATOS}

Los datos han sido organizados en ocho categorías que describen las variables didácticas del proceso de enseñanza-aprendizaje. Cada categoría refiere a las creencias que los informantes sostienen respecto a: el estudiante, el aprendizaje en general, el aprendizaje de los estudiantes de las áreas de Trabajo Social y Periodismo, el docente, el plan de estudios y los contenidos de enseñanza, la metodología de enseñanza, los objetivos de aprendizaje y la evaluación de dichos aprendizajes.

\begin{tabular}{|c|c|}
\hline Dimensión & Categoría 1 \\
\hline $\begin{array}{c}\text { Creencias respecto al proceso } \\
\text { de enseñanza-aprendizaje }\end{array}$ & El estudiante de educación superior \\
\hline
\end{tabular}

En cuanto a la dimensión creencias respecto al proceso de enseñanza-aprendizaje, los informantes entrevistados mencionaron bastantes elementos en común para la categoría creencias respecto al estudiante de educación superior y las características que debería tener un estudiante de las carreras de Periodismo y Trabajo Social. Los informantes coincidieron en que los estudiantes deberían tener habilidades sociales, una buena autoestima y una actitud reflexiva, como lo evidencian los siguientes ejemplos:

Informante 1 (Periodismo): [el estudiante de Trabajo Social necesita] “...habilidades sociales... alta capacidad de comunicación..."

Informante 2 (Periodismo): “.... [el estudiante de Trabajo Social necesita] una personalidad bastante definida... no puede ser tímido..."

Informante 3 (Trabajo Social): “... [el estudiante de Trabajo Social necesita] Buena autoestima, no pueden ser personas con problemas de salud mental..."

Informante 4 (Trabajo Social): “... [el estudiante de Trabajo Social tiene que ser] una persona capaz de realizar un análisis crítico, [tiene que ser] alguien reflexivo...”

Los informantes de Periodismo consideran importante que el estudiante que ingresa a estudiar Periodismo tiene que poseer fuertes habilidades comunicativas y seguridad en sí mismo, para así poder desempeñarse exitosamente y tener un espacio en el mundo laboral.

En el caso de los informantes de Trabajo Social, sus creencias sostienen que el estudiante que estudia Trabajo Social debe tener una habilidad comunicativa muy desarrollada, y a su vez, debe ser capaz de formular juicios críticos, a partir de la reflexión. Esto, según la literatura teórica promueve el desarrollo del pensamiento crítico, ayudando a que los estudiantes desarrollen la capacidad de valorar y mejorar las situaciones que vivencian. El resto de los informantes del área de Trabajo Social le atribuyen mayor importancia al hecho de que el estudiante no presente ninguna patología psicológica. 


\begin{tabular}{|c|c|}
\hline Dimensión & Categoría 2 \\
\hline $\begin{array}{c}\text { Creencias respecto al proceso } \\
\text { de enseñanza-aprendizaje }\end{array}$ & El aprendizaje \\
\hline
\end{tabular}

Al ser preguntados los diez sujetos informantes por sus creencias con respecto al aprendizaje, todos poseen una conceptualización distinta de éste, pero coinciden en algunos puntos. Hay cinco docentes que coinciden en el hecho de que el aprendizaje es un proceso de construcción continuo a lo largo de la vida como, por ejemplo, lo indican los siguientes fragmentos:

Informante 2 (Periodismo): “... [el aprendizaje es] como un proceso, es construir conocimientos e instrumentos..."

Informante 6 (Trabajo Social): “... [el aprendizaje] no se acaba, es algo que está en constante construcción..."

Informante 5 (Periodismo): “... [el aprendizaje es] como un proceso lento, que no puede lograrse de un día para otro..."

Los informantes de Trabajo Social incorporan un nuevo elemento a lo mencionado por los informantes de Periodismo; dicho componente se relaciona con poner en práctica lo aprendido, es decir, llevar a la acción el nuevo conocimiento, competencia o actitud aprendida.

Informante 3 (Trabajo Social): “... [aprendizaje] es cuando tú adquieres un nuevo conocimiento, eres capaz de desarmarlo, manejarlo, trabajarlo y aplicarlo..."

Informante 4 (Trabajo Social): “... [aprendizaje son] las distintas formas que nosotros, las personas [tenemos] de captar cierta información y luego [poder] ponerla en práctica...”

Agrega uno de los informantes que el aprendizaje es un proceso de interacción, un proceso más dialógico, más horizontal entre aquél que entrega el conocimiento como aquél que lo recibe, según el fragmento siguiente:

Informante 1 (Periodismo): “... [el aprendizaje] lo definiría como un proceso de interacción, más que un proceso lineal o vertical de enseñanza, yo lo definiría más como un proceso de interacción, de construcción entre el que entrega conocimiento y el que recibe...”

\begin{tabular}{|c|c|}
\hline Dimensión & Categoría 3 \\
\hline $\begin{array}{c}\text { Creencias respecto al proceso de } \\
\text { enseñanza-aprendizaje }\end{array}$ & $\begin{array}{c}\text { El aprendizaje de los estudiantes de } \\
\text { Trabajo Social y Periodismo }\end{array}$ \\
\hline
\end{tabular}


Al ser preguntados los informantes acerca de las creencias que ellos sostenían, respecto a cómo aprendían sus estudiantes, ambos grupos, Trabajo Social y Periodismo, declararon situaciones bastante similares como, por ejemplo, que sus estudiantes no sabían cómo estudiar, y que sólo llegaban a un nivel de memorización, como lo evidencian las siguientes respuestas.

Informante 6 (Trabajo Social): “...mis estudiantes estudian mal, estudian de memoria...”

Informante 10 (Trabajo Social): "mis estudiantes utilizan el...aprendizaje de memoria..."

La mayoría de los informantes declaran que sus estudiantes llegan a la educación terciaria sin hábitos de estudios, sin las competencias básicas necesarias requeridas para comenzar su proceso de formación. Esto queda en evidencia al declarar que:

Informante 3 (Trabajo Social): “...[mis estudiantes son] malos lectores, tienen mala comprensión lectora, se requiere de mucha ejemplificación concreta..."

Informante 4 (Periodismo): “...Yo creo que los alumnos no tienen un sistema de aprendizaje, ellos no vienen con un sistema de aprendizaje..."

Un informante de la carrera de Trabajo Social utiliza un componente más dialógico con sus estudiantes, que lo obliga a ejemplificar y contextualizar ampliamente la información que se trabaja en clases. Es así como lo declara en la entrevista:

Informante 3 (Trabajo Social): “...Yo converso harto con ellos, les muestro lo que dicen los autores, siempre ejemplifico, siempre los estoy llevando a la vida personal, así logran entender lo que dice el autor..."

Tres informantes de Periodismo tienen una mirada distinta con respecto a cómo aprenden sus estudiantes. La razón es que Periodismo es una carrera con un alto componente práctico. Esto hace que los informantes apunten a desarrollar competencias que sus estudiantes tienen que poner en práctica rápidamente; es por esta razón que ellos creen que sus estudiantes aprenden mediante la experiencia como lo demuestran las siguientes citas tomadas de la entrevista:

Informante 2 (Periodismo): “... [los estudiantes] aprenden mucho por experiencia...”

Informante 5 (Periodismo): “...Yo trato de que ellos [los estudiantes] aprendan haciendo las cosas..."

Informante 8 (Periodismo): “...[yo creo que los estudiantes aprenden] a porrazo limpio...” 


\begin{tabular}{|c|c|}
\hline Dimensión & Categoría 4 \\
\hline $\begin{array}{c}\text { Creencias respecto al proceso de } \\
\text { enseñanza-aprendizaje }\end{array}$ & Las características de un buen docente \\
\hline
\end{tabular}

En relación con la categoría creencias respecto a ser un buen docente, las respuestas generadas por el grupo de informantes fueron variadas, pero se logran destacar tres grupos; en el primer grupo están quienes se centran en el logro satisfactorio de los objetivos de enseñanza. El segundo grupo considera relevante la motivación de parte del docente y la cercanía que éste tenga con sus estudiantes y un tercer grupo, con una visión más holística, considera el área afectiva y un sólido dominio del conocimiento por parte del docente. En los fragmentos extraídos de la entrevista se evidencian las creencias de aquellos docentes que consideran importante el logro de los objetivos:

Informante 2 (Periodismo): “...[un buen docente es] el que obtiene mejores resultados...”

Informante 6 (Trabajo Social): “... [un buen docente es] aquel que entrega [los] conocimientos que tiene que entregar..."

Informante 3 (Trabajo Social): “... [un buen docente] tiene la capacidad de poder adecuar los objetivos de aprendizaje a las necesidades de los alumnos..."

Estos informantes basan sus creencias de ser un buen docente en el hecho de que éste sea capaz de lograr los mejores resultados o cumplir con los objetivos de enseñanza propuestos. Logran los informantes incorporar un aspecto fundamental en la docencia, a saber, el hecho de que todos los estudiantes aprendan y desarrollen las competencias necesarias, y que éstas estén de acuerdo a las necesidades de los estudiantes, lo que implica conocerlos bien.

Para el segundo grupo, un buen docente es quien logra motivar e inquietar a sus alumnos por el conocimiento y logra que todos sus estudiantes adquieran las competencias propuestas en los programas de estudios, tal es el caso de:

Informante 8 (Periodismo): “... [un buen docente es] el que motiva a los alumnos a aprender más de la cuenta, el que logra que todos sus alumnos aprendan e interioricen ese aprendizaje..."

Informante 6 (Trabajo Social): “...[un buen docente es] un gallo que inquiete a los alumnos, que motive, que sea motivador..."

Informante 3 (Trabajo Social): “... [un buen docente es el que logra] que los alumnos se interesen, yo creo que [para] mí esa es la clave..."

El tercer grupo de informantes aporta más características respecto a lo que es ser un buen docente. Además de poseer una experticia en la disciplina que enseña, un buen docente debe poseer habilidades motivacionales y afectivas que favorezcan el proceso de enseñanza-aprendizaje. 
Informante 9 (Periodismo): “... [un buen docente debe tener] una base de conocimiento, experiencia en investigación, que sepa escuchar, que sea mitad psicólogo y mitad académico y sobre todo valores..."

Informante 2 (Periodismo): “...[un buen docente es] una persona que tiene vocación, que le guste enseñar, que se preocupe por investigar, [por hacer] más eficiente el proceso de enseñanza-aprendizaje

El tercer grupo tiene una mirada más holística de lo que es un buen docente a diferencia de los dos grupos mencionados anteriormente. Se observa que este grupo considera aspectos tales como: la experticia de la disciplina que enseña, experiencia en investigación y vocación por la docencia.

\begin{tabular}{|c|c|}
\hline Dimensión & Categoría 5 \\
\hline $\begin{array}{c}\text { Creencias respecto al proceso } \\
\text { de enseñanza-aprendizaje }\end{array}$ & $\begin{array}{c}\text { El plan de estudios y los contenidos } \\
\text { de enseñanza }\end{array}$ \\
\hline
\end{tabular}

Con respecto a la categoría creencias respecto al plan de estudios y los contenidos de enseñanza, se encuentran dos tendencias en ambos grupos de estudio. Existen informantes que creen que hay contenidos de mayor relevancia que otros y que la teoría tiene gran importancia en el proceso de enseñanza, tal como lo demuestran las siguientes citas, tomadas de la entrevista semiestructurada:

Informante 3 (Trabajo Social): "Yo siempre digo que la metodología de caso es la más importante..." [ refiriéndose a la asignatura que ella enseña]

Informante 7 (Trabajo Social): “...Sí, hay temas más importantes, bases teóricas o conceptuales...[más importantes]..."

Informante 1 (Periodismo): “...El alumno tiene que saber muchos contenidos audiovisuales...” [refiriéndose al área que él enseña]

Tanto para algunos informantes del área de Trabajo Social como para algunos de Periodismo existen contenidos más importantes que otros en el plan de estudios de las respectivas carreras, coincidiendo en que las asignaturas que ellos imparten son las de mayor importancia dentro del plan de estudios y dentro de estas asignaturas algunos contenidos por sobre otros, dando prioridad a la teoría por sobre la práctica.

Dentro de la misma categoría se encuentra otro grupo de docentes que tiene una mirada distinta del plan de estudios y de los contenidos que se presentan en éste, declarando que todos los contenidos son importantes, como lo demuestran los siguientes fragmentos:

Informante 1 (Periodismo): “...Todos los contenidos que están considerados dentro del programa son fundamentales..."

Informante 4 (Trabajo Social): “...Yo creo que son todos (los contenidos) importantes...” 
Informante 7 (Trabajo Social): “....No siento que una (asignatura) tenga mayor importancia que otra..."

Con respecto a la categoría anterior se observa que existen dos tendencias en este grupo de informantes, los que declaran que existen contenidos y asignaturas más relevantes e importantes que otras en la formación de los futuros profesionales de las áreas de Periodismo y Trabajo Social, y un segundo grupo que cree que las asignaturas y contenidos que están presentes en el plan de estudios son todos relevantes.

\begin{tabular}{|c|c|}
\hline Dimensión & Categoría 6 \\
\hline $\begin{array}{l}\text { Creencias respecto al proceso } \\
\text { de enseñanza-aprendizaje }\end{array}$ & La metodología de enseñanza \\
\hline
\end{tabular}

Con respecto a la categoría creencias respecto a la metodología de enseñanza, o métodos utilizados, por los informantes para llevar exitosamente la transposición didáctica, entendida como el hacer enseñable el saber disciplinario, el grupo de informantes declara tener una diversidad de metodologías de enseñanza, destacando el trabajo en equipo, los trabajos prácticos y las clases expositivas como es el caso de los siguientes informantes:

Informante 9 (Periodismo): “...Explicar conceptos, definiciones, casos y la discusión...[son metodologías que utilizo]"

Informante 8 (Periodismo): “ ...En las [asignaturas] teóricas no hay mucha opción metodológica; Yo estoy ahí y trato de explicarles la materia de la forma más clara para ellos..."

Informante 1 (Periodismo): “...Bueno, voy mezclando clases, hay fases expositivas y prácticas y hay otras que son mixtas..."

Los tres docentes recién mencionados tienen un fuerte componente teórico en sus asignaturas; por lo tanto, sus clases son fundamentalmente teóricas y el foco de las clases está basado en el docente más que en los estudiantes, lo que da cuenta de una metodología centrada en el docente.

Un caso distinto dentro de la misma categoría lo constituyen los informantes de la carrera de Periodismo, que por las capacidades que intentan desarrollar en sus estudiantes presentan clases más prácticas, donde es necesario que el estudiante elabore un producto final.

Informante 5 (Periodismo): “...es el método práctico fundamentalmente... [el que utilizo en mis clases]"

Un informante de la carrera de Periodismo que está a cargo del área audiovisual, que tiene un fuerte componente tecnológico y computacional, declara que sus metodologías son "prácticas" debido a que el estudiante debe poner inmediatamente en práctica lo que está 
aprendiendo. En términos pedagógicos, esto se denomina praxis, momento en el cual una teoría se convierte en parte de la experiencia del estudiante. En otras palabras, las teorías no son solamente absorbidas a nivel intelectual en un aula, las ideas son experimentadas en el mundo real, y luego seguidas de una contemplación reflexiva. De este modo, los conceptos se conectan con la experiencia y el aprendizaje se transforma en significativo.

Informante 8 (Periodismo): “...Mucha dinámica en clases, mucho trabajo práctico [es el método que utilizo en mis clases]"

Como se puede inferir de lo declarado por estos informantes, sus asignaturas tienen un fuerte componente práctico en donde sus estudiantes deben presentar resultados concretos, productos finales, más que demostrar sólo la adquisición de conocimientos.

Dentro de la misma categoría creencias respecto a la metodología de enseñanza, el grupo de informantes de la carrera de Trabajo Social también declaran tener metodologías mixtas, que combinan las clases expositivas, las lecturas, los trabajos grupales y la discusión, tal como se observa en los siguientes fragmentos:

Informante 10 (Trabajo Social): “...Yo [utilizo] harto trabajo en equipo, harto trabajo participativo..."

Informante 4 (Trabajo Social): “... [Yo utilizo] exposiciones, apoyo audiovisual, lectura, trabajos grupales y discusión..."

Informante 3 (Trabajo Social): “...Los trabajos grupales, lecturas colectivas...presentaciones grupales [son mis metodologías preferidas]..."

Como se puede observar en el grupo de Trabajo Social, quienes trabajan con un fuerte componente teórico, utilizan el trabajo en equipo y la discusión como metodologías de enseñanza, con la idea de formar un profesional reflexivo que además sea capaz de integrarse fácilmente a un grupo de trabajo multidisciplinario.

\begin{tabular}{|c|c|}
\hline Dimensión & Categoría 7 \\
\hline $\begin{array}{l}\text { Creencias respecto al proceso } \\
\text { de enseñanza-aprendizaje }\end{array}$ & Los objetivos de aprendizaje \\
\hline
\end{tabular}

La mayoría de los informantes tienen creencias bastante claras con respecto a los objetivos de aprendizaje y sostienen que ellos son guías orientadoras del accionar pedagógico; así lo declaran, por ejemplo, las siguientes citas:

Informante 1 (Periodismo): “...[los objetivos] ayudan a planificar las actividades de enseñanza, por lo mismo están en constante modificación..."

Informante 1 (Periodismo): “...hay que saber para dónde nos vamos a orientar...” 
Informante 5 (Periodismo): “...Yo creo que [son] muy importantes, porque en la medida en que uno tiene claro los objetivos, puede desarrollar las actividades [de aprendizaje]..."

Los informantes de la carrera de Trabajo Social incorporan el proceso de evaluación de los aprendizajes, declarando que para ellos los objetivos son guías orientadoras de todo el proceso de enseñanza-aprendizaje, aun más, los objetivos son guías que les permiten orientar los procesos de evaluación, como lo ilustran los siguientes fragmentos:

Informante 3 (Trabajo Social): “...[los objetivos] me permiten orientar la organización de los temas, pensar y diseñar los procesos de evaluación..."

Informante 4 (Trabajo Social): “...[los objetivos] son los que me orientan la evaluación, son los que me determinan el proceso a seguir..."

Al preguntar a los docentes si ellos comunicaban los objetivos de enseñanza, la mayoría declaró que lo hacían pero sólo al momento de leer el programa, es decir, al comienzo de la asignatura, como lo demuestra la siguiente cita:

Informante 4 (Trabajo Social): “... Mira, es que uno le pone objetivos al programa y después uno nunca más los va a ver...”

Los informantes, en su mayoría declaran los objetivos de los programas de las asignaturas que enseñan al comienzo del semestre, cuando leen el programa en conjunto con los estudiantes, pero después en pocas oportunidades revisan nuevamente los objetivos en el aula. A su vez, señalan que no declaran objetivos antes de cada clase.

\begin{tabular}{|c|c|}
\hline Dimensión & Categoría 8 \\
\hline Creencias respecto al proceso de enseñanza & La evaluación de los aprendizajes \\
\hline
\end{tabular}

Indudable es el hecho de que la evaluación tiene un rol protagónico y fundamental en todo el proceso didáctico. La mayoría de los informantes encuestados consideran la evaluación como un proceso de verificación de la adquisición de los contenidos entregados durante el proceso de enseñanza-aprendizaje, tal como lo demuestran las siguientes citas:

Informante 8 (Periodismo): “...[la evaluación es] la manera de comprobar que ciertos contenidos se hayan interiorizado en los alumnos..."

Informante 1 (Periodismo): “...según yo [la evaluación] es corrección...”

Informante 2 (Periodismo): “... [la evaluación sirve] para saber si los alumnos han desarrollado el aprendizaje..." 
Informante 10 (Trabajo Social): “...[la evaluación] es la forma que yo tengo para saber si los chiquillos adquirieron los conocimientos o no..."

Informante 4 (Trabajo Social): “...[la evaluación sirve] para poder constatar los niveles de aprendizaje de los estudiantes..."

Los cinco informantes citados anteriormente sólo utilizan la evaluación como una herramienta para comprobar cuánto han logrado aprender sus estudiantes. Es una herramienta utilizada al final de un periodo específico. También declaran utilizar sólo instrumentos evaluativos tradicionales tales como; certámenes, tests, controles de lectura. Por lo general consideran dos certámenes y el examen final en su proceso didáctico; así lo demuestran las siguientes citas.

Informante 7 (Trabajo Social): “...[utilizo] los certámenes, tests, los trabajos de investigación, informes de aplicación...”

Informante 4 (Trabajo Social): “...Yo hago certámenes y varios ejercicios prácticos...”

Informante 3 (Trabajo Social): “...[realizo] dos certámenes, un examen, tres o cuatro evaluaciones complementarias que son controles de lecturas..."

En la categoría creencias respecto a la evaluación, algunos informantes de Periodismo tienen una visión similar a los informantes de Trabajo Social, al ver la evaluación como una herramienta de medición; sin embargo, en el área de Periodismo algunos informantes buscan medir las competencias de los estudiantes mediante productos finales. El fundamento de medir las competencias por medio de actividades prácticas se basa en las asignaturas que estos docentes enseñan, puesto que ellas están asociadas al uso de las nuevas Tecnologías de la Información y Comunicación.

Informante 2 (Periodismo): “...[para evaluar] Yo siempre pido un producto final...”

Informante 1 (Periodismo): “...[para evaluar] Yo utilizo trabajos prácticos, creo que sólo aplico una prueba teórica en el año..."

Informante 7 (Periodismo): “...[para evaluar] Les hago ejercicios prácticos...”

Algunos informantes declaran que el único instrumento evaluativo que ellos pueden utilizar para medir las competencias de sus estudiantes son los trabajos prácticos, puesto que ellos son el único modo de constatar si los estudiantes realmente desarrollaron las competencias propuestas al inicio del programa. 


\section{CONCLUSIONES}

Los análisis presentados anteriormente han sido abordados, fundamentalmente, desde el punto de vista de la tríada didáctica que en su esencia reconoce tres variables fundamentales del proceso didáctico: docente, discente y contenidos.

Aunque los informantes de Trabajo Social y Periodismo provienen de áreas disciplinarias que no consideran formación pedagógica para sus estudiantes durante su paso por la universidad, resulta interesante constatar que ciertos informantes conceptualizan el aprendizaje como un proceso de construcción e interacción constante entre los actores involucrados en dicho proceso. También merece atención la idea de que el estudiante logra un aprendizaje cuando éste es capaz de construir, reconstruir, manejar, trabajar y aplicar dicho aprendizaje.

Los informantes, ciertamente, muestran una noción de aprendizaje que se acerca a acepciones más cognitivas que enfatizan el rol del estudiante que posee herramientas cognitivas que le permiten alcanzar aprendizajes más permanentes y con potencial de ser aplicados a nuevas situaciones de aprendizaje.

La proveniencia de los informantes del área humanista se observa nítidamente al momento que ellos indican que el estudiante de enseñanza superior que desea estudiar Periodismo o Trabajo Social debe poseer habilidades sociales, analíticas y comunicativas que le permitan desempeñarse eficientemente en el mercado laboral.

La gran mayoría de los informantes, por otro lado, considera que sus estudiantes no saben cómo aprender y utilizan la memorización como estrategia principal de aprendizaje. Evidencian las pobres habilidades de comprensión de lectura de los estudiantes y, a su vez, corroboran el hecho de que el estudiante aprende haciendo.

El logro de aprendizajes de calidad por parte de los estudiantes requiere, sin duda, de un esfuerzo particular por parte del docente. En este sentido un buen docente, para los informantes, es el que motiva, inquieta e interesa a los estudiantes. También, un buen docente es capaz de obtener los mejores resultados de aprendizaje, de adecuar los objetivos de aprendizaje, de escuchar a los estudiantes. En definitiva, un buen docente posee una base de conocimiento y experiencia investigativa que puede aplicar en su práctica pedagógica.

Los contenidos de enseñanza para algunos informantes son todos importantes; otros consideran que existen algunos más relevantes que otros. Las metodologías utilizadas para trasponer dichos contenidos son variadas, señalan los informantes. Mencionan el estudio de casos, la explicación de conceptos, la discusión, las clases prácticas, las dinámicas grupales, el trabajo en equipo, las lecturas y las sesiones audiovisuales como estrategias metodológicas que les permiten lograr aprendizajes.

Consultados respecto a los objetivos de enseñanza, muchos informantes les atribuyen una función orientadora relacionada con: el diseño de actividades, la selección de contenidos y la elaboración de instrumentos evaluativos.

En la revisión realizada hasta el momento los informantes revelan creencias respecto a su praxis pedagógica muy acordes con los principios de una muy buena enseñanza; sin embargo, al ser consultados respecto a la evaluación, casi todos señalan que ésta sirve para comprobar lo que aprendieron los estudiantes. Uno de ellos, además, enfatiza que la evaluación es sinónimo de corrección. 
Llama la atención que informantes tan conscientes de las buenas prácticas de enseñanza, por lo menos a nivel discursivo, debiliten sus respuestas al focalizar sus perspectivas en solo una de las funciones de la evaluación, esto es, la comprobación de aprendizajes.

No hay indicios de procesos de autoevaluación o coevaluación por parte de los estudiantes. No se observan, tampoco, indicios de procedimientos evaluativos más alternativos como el uso del portafolio, los diarios autobiográficos o las entrevistas.

Los resultados de este proyecto Fondecyt, sumados a otros proyectos realizados por los autores, permiten establecer, con cierto grado de precisión, algunos puntos abiertos a la discusión de los lectores:

1. Las creencias de los informantes a nivel discursivo denotan una cercanía con prácticas de enseñanza que deberían lograr aprendizajes de calidad por parte de los estudiantes.

2. La experiencia académica y el contexto universitario de los informantes, caracterizados por actividades de docencia, de investigación y de extensión, hacen que los informantes posean creencias que se acercan a una literatura científica actualizada.

3. El desafío mayor de esta investigación, ciertamente no trabajado en este artículo, es comprobar que las creencias que los docentes sostienen a nivel discursivo guardan congruencia con el nivel de su actuación en el aula.

Parece ser que es en este último punto donde las creencias de los informantes presentan un mayor grado de complejidad, puesto que la claridad discursiva respecto a algunas variables del proceso de enseñanza-aprendizaje no se visualiza con la misma claridad en el plano de la actuación en el aula.

La enseñanza es un proceso multidimensional y complejo. El docente que tiene un conocimiento pedagógico y disciplinar más profundo y una conciencia mayor sobre los diferentes componentes y variables de la enseñanza-aprendizaje, está mejor preparado para realizar juicios y tomar decisiones apropiadas respecto de dicho proceso.

Aunque la experiencia es un componente clave para el desarrollo profesional docente, por sí sola es insuficiente como base para el crecimiento profesional. Muchos aspectos de la enseñanza ocurren en el día a día, y los docentes desarrollan rutinas y estrategias para manejar estas dimensiones recurrentes de la enseñanza. Sin embargo, la investigación sugiere que, para los docentes experimentados, muchas rutinas y estrategias pedagógicas son aplicadas casi automáticamente y no implican reflexión alguna. La experiencia es el punto inicial para el desarrollo docente, pero para que ésta tenga un rol productivo es necesario examinar dicha experiencia de manera sistemática.

En este sentido, la reflexión crítica implica el examen de las experiencias docentes como base para la autoevaluación y la toma de decisiones y como una fuente importante para la innovación educativa. Esta reflexión implica preguntarse acerca de cómo y por qué las cosas son de la forma que son, qué sistemas valóricos ellas representan, qué alternativas pueden estar disponibles y qué limitantes existen cuando las cosas se hacen de una manera y no de otra.

Estas conclusiones reflejan que si los académicos están involucrados activamente en la reflexión de lo que sucede en sus aulas, ellos están en posición de descubrir si existe alguna brecha entre lo que enseñan y lo que los estudiantes aprenden. La premisa fundamental de la investigación de los procesos de enseñanza-aprendizaje es, sin duda, 
que los docentes deberían utilizar sus aulas como laboratorios para estudiar el proceso de aprendizaje. Los docentes deberían convertirse en observadores expertos y sistemáticos acerca de cómo sus estudiantes aprenden. Si se trata de profundizar en el fenómeno de las creencias y actuaciones pedagógicas de los docentes, se debe profundizar en los significados que ellos otorgan al proceso de enseñanza-aprendizaje desde su experiencia en el marco del contexto universitario.

\section{BIBLIOGRAFIA}

Andrés, G. y P. Echeverri (2001). Pensamiento docente y práctica pedagógica. Una investigación sobre el pensamiento práctico de los docentes. Bogotá: Magisterio.

Andrews, S. (2003). Just like instant noodles': L2 teachers and their beliefs about grammar pedagogy. Teachers and Thinking: Theory and Practice 9: 351-370.

Appel, J. (1995). Diary of a language teacher. Oxford: Heinemann.

Bailey K., A. Curtis \& D. Nunan (2001). Pursuing professional development. Boston: Newbury House. Teacher Development.

Ben-Peretz, M. (2002). Retired teachers reflect on learning from experience. Teachers and Thinking: Theory and Practice 8: 312-324.

Biddle, B., T. Good y I. Goodson (2000). La enseñanza y los profesores I. La profesión de enseñar. Buenos Aires: Paidós.

Borg, S. (2003). Teacher cognition in language teaching: a review of research on what language teachers think, know, believe, and do. The International Abstracting Journal for Language Teachers and Applied Linguists 36: 81-109.

Cárdenas, A., A. Rodríguez y R. Torres (2000). El maestro, protagonista del cambio educativo. Bogotá: Magisterio.

Camps, A. (2001). El aula como espacio de investigación y reflexión. Investigaciones en didáctica de la lengua. Barcelona: Graó.

Carusetta, E. \& P. Cranton (2005). Nurturing authenticity: a conversation with teachers. Teaching in Higher Education 10: 285-297.

Crookes, G. (2003). A practicum in Tesol. Professional development through teaching practice. Cambridge: Cambridge Language Education.

Frabboni, F. (2001). El libro de la pedagogía y la didáctica I: la educación. Madrid: Editorial popular.

Freeman, D. (2002). The hidden side of the work: Teacher knowledge and learning to teach. A perspective from North American educational research on teacher education in English language teaching. Language Teaching. The International Abstracting Journal for Language Teachers and Applied Linguists 35: 1-13.

Gelhard, J. \& R. Oprandy (1999). Language teaching awareness. A guide to exploring beliefs and practices. Cambridge: Cambridge University Press.

Gonzáles S., E. Río y S. Rosales (2001). El Currículum oculto en la escuela. Buenos Aires: Lumen Humanitas.

Goodson, I. \& U. Numan (2002). Teacher's life worlds, agency and policy contexts. Teachers and Thinking: Theory and Practice 8: 269-277.

Hamel, F. (2003). Teacher understanding of student understanding: Revising the gap between teacher conceptions and students' ways with literature. Research in the Teaching of English 38: 49-84.

Hashweh, M. (2005). Teacher pedagogical constructions: a reconfiguration of pedagogical content knowledge. Teachers and Thinking: Theory and Practice 11: 273-292. 
Johnson, K. \& P. Golombek (2002). Teachers' narrative inquiry as professional development. Cambridge: Cambridge University Press.

Johnstone, R. (2000). Research on language learning and teaching: 1999. Language Teaching. The International Abstracting Journal for Language Teachers and Applied Linguists 33: 141-162.

Kansanen, P., K. Tirri, M. Meri, I. Krokfors, L., Husu, J. \& R. Jyrhämä (2000). Teachers' pedagogical thinking. New York: American University Studies.

Libedinsky, M. (2001). La innovación en la enseñanza. Buenos Aires: Paidós.

Menin, O. (2001). Pedagogía y Universidad. Currículo, didáctica y evaluación. Santa Fe: Ediciones Homosapiens.

Muchmore, J. (2004). A teachers' life. San Francisco: Backalong books.

Richard-Amato, P. (2003). Making It happen. New York: Longman.

Sanjurjo, L. (2002). La formación práctica de los docentes. Reflexión y acción en el aula. Santa Fe: Homosapiens.

Tsui, A. (2003). Understanding expertise in teaching. Cambridge: Cambridge University Press.

Woods, D. (1996). Teacher cognition in language teaching: beliefs, decision-making and classroom practice. Cambridge: Cambridge University Press. 